\title{
Intensive Care Medicine in 2050: toward an intensive care unit without waste
}

\author{
George L. Anesi ${ }^{1,2,3,4^{*}}$, Jason Wagner ${ }^{1,5}$ and Scott D. Halpern ${ }^{1,2,3,4,6}$
}

๑ 2016 Springer-Verlag Berlin Heidelberg and ESICM

In the year 2050 we will unambiguously reimburse healthcare based on value [1], and so there is good reason to suspect that we will have targeted and reduced many services that provide little or no benefit to patients. Because it is impossible to prove that an intervention provides zero benefit for all conceivable patients, it is useful to consider ways to reduce both no-value care and low-value care (e.g., services that provide small benefits, but come at a high cost).

There are two main categories of low-value critical care: (1) the allocation of intensive care unit (ICU) beds to patients who will not benefit over admission to a ward, and (2) the provision of excessive critical care resources to patients who appropriately gain entry into the ICU. While both are important, avoiding low-value ICU admissions portends generally greater cost reductions than avoiding waste from low-value services delivered to patients already admitted to the ICU [2-4].

A high-value ICU, therefore, will strive to admit only patients who will benefit from critical care services, and will maximize the value of care delivery to those who are admitted. Optimizing triage decisions for potential ICU admissions is an emerging and challenging area of research. This will require innovative study designs that allow for quasi-experimental, if not fully randomized, comparisons of outcomes of ward and ICU patients admitted from emergency departments. Such work will require highly granular adjustment for hospital- and patient-level risk factors. Ultimately, it may yield personalized triage approaches that are based not only on such formative work but also incorporate genetic predispositions [5] and real-time biomarkers [6] to help predict

\footnotetext{
*Correspondence: george.anesi@uphs.upenn.edu

${ }^{1}$ Division of Pulmonary, Allergy, and Critical Care Medicine, Hospital of the University of Pennsylvania, 3600 Spruce Street, Gates Building, Room GA 5044, Philadelphia, PA 19104, USA

Full author information is available at the end of the article
}

individual risk of clinical decompensation and ultimately "net ICU benefits".

To date, the literature has not been up to this formidable challenge. Several studies suggest that patients who may not benefit from ICU admission are nonetheless commonly admitted to ICUs. These groups include patients with diabetic ketoacidosis, non-massive pulmonary emboli, and heart failure not requiring mechanical ventilation [7]. On the other hand, one recent study-the first to use a quasi-experimental design by incorporating an instrumental variable-identified a potential benefit to ICU admission for low-risk older adults with pneumonia [8]. However, it is difficult to draw practice-relevant conclusions from even this rigorous study because of potential confounding by unmeasured differences among hospitals (its design addressed whether admission to one hospital's ICU yields better outcomes than admission to another hospital's ward).

Other data suggest there are also patients who are admitted to ICUs when aggressive care will be insufficient to meaningfully forestall death [9]. While mortality is an important outcome, many factors other than survival should also be considered when studying optimal ICU bed allocation such as hospital length of stay, effective symptom palliation, and caregiver bereavement outcomes. If preference-concordant palliation can be better achieved in some ICUs than on corresponding hospitals' wards, ICU admission for this purpose may be valuable indeed.

Another important question to ask in an effort to reduce waste is when has a critically ill patient recovered sufficiently for a safe ICU discharge. ICU resources delivered to a patient after critical care is no longer considered necessary are wasteful [10]. Additionally, interventions that may shorten ICU length of stay without improving mortality may nonetheless serve the goals both of patients and families (by shortening uncomfortable ICU stays) and of society (by reducing costs). Thus, future

\section{Springer}




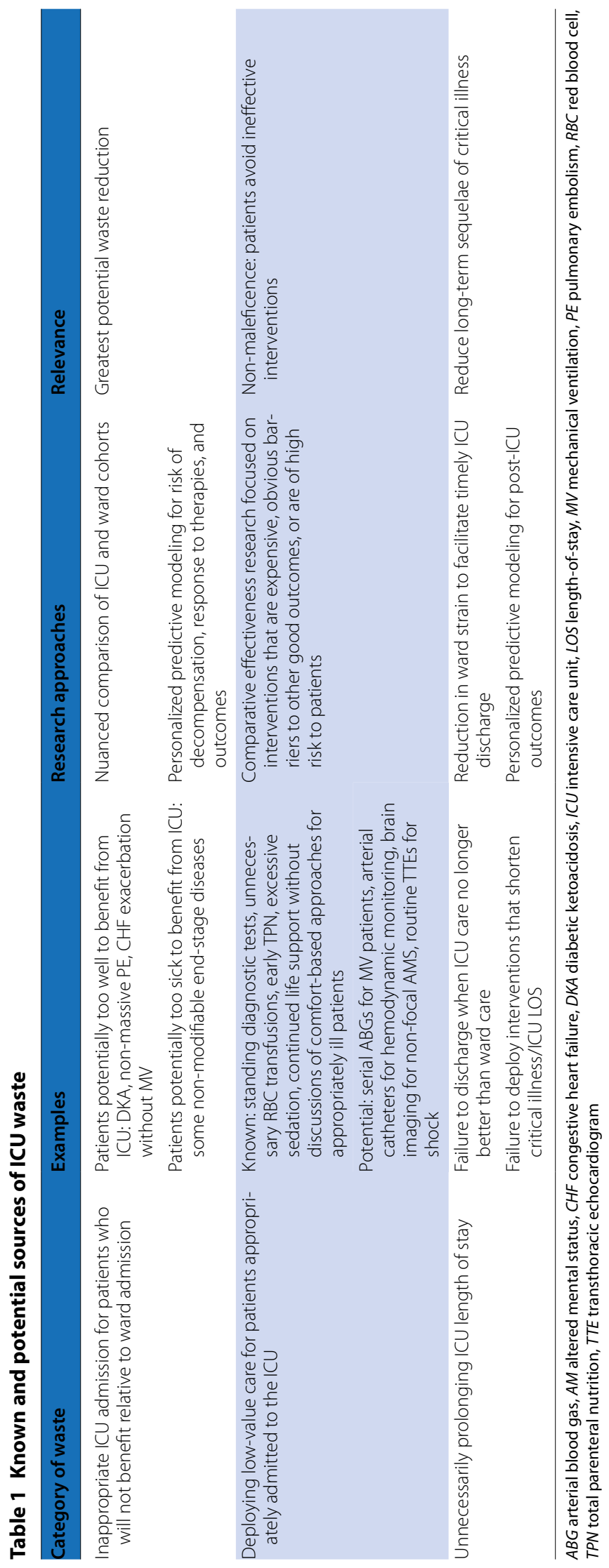


ICUs that more routinely perform daily sedation interruption and spontaneous breathing trials [11] and more commonly administer corticosteroids in septic shock [12] will reduce wasteful time in the ICU.

Aside from efforts to improve ICU triage decisions, there is also strong motivation to promote non-maleficence by eliminating ICU care that is burdensome without improving morbidity or mortality. Comparative effectiveness research, such as that which informed the Critical Care Collaborative's Choosing Wisely list [2], seeks to identify high-value diagnostic and therapeutic interventions to reduce wasteful practices. In addition to the Choosing Wisely list, there are many additional interventions for which the harms or costs may outweigh the benefits. Future efforts to evaluate, and potentially reduce, utilization of diagnostics such as serial arterial blood gas measurements, peripheral arterial catheters for hemodynamic monitoring [13], brain imaging in the absence of focal neurological deficits, and routine echocardiography may therefore further improve the value of critical care (Table 1).

As with any paradigmatic shift, implementation is as vital as discovery. Data predicting which patients would benefit from an ICU admission, for example, would need to be immediately and inexpensively available to clinicians making real-time triage decisions. Similar data to inform advance care planning before the onset of critical illness could further improve the efficiency of critical care.

Finally, we must fully embrace the importance of educating our next generation of ward and critical care providers to lead in practicing high-value care [14]. More generally, we must engage society in open dialogue about how such changes in our approach will be evidence based, ethically sound, and equitably applied. While still nascent in their approach to integrating value with medical education, the Accreditation Council for Graduate Medical Education in the USA now includes safety, quality, and cost-effectiveness as mandatory training milestones that encompass medical professionalism by serving both individual patients and society [15]. To succeed in 2050, medical professionals must recognize their own cognitive biases, train future clinicians as responsible stewards of resources, and devise evidence-based strategies to efficiently de-adopt wasteful practice modalities that impair our ability to deliver high-quality critical care [16-18].

\footnotetext{
Author details

${ }^{1}$ Division of Pulmonary, Allergy, and Critical Care Medicine, Hospital of the University of Pennsylvania, 3600 Spruce Street, Gates Building, Room GA 5044 Philadelphia, PA 19104, USA. ${ }^{2}$ Department of Biostatistics and Epidemiology, Center for Clinical Epidemiology and Biostatistics, University of Pennsylvania, Philadelphia, PA, USA. ${ }^{3}$ Acute Care Health Services Research Group, University of Pennsylvania, Philadelphia, PA, USA. ${ }^{4}$ Leonard Davis Institute of Health Economics, University of Pennsylvania, Philadelphia, PA, USA. ${ }^{5}$ Corporal Michael J.
}

Crescenz Veterans Affairs Medical Center, Philadelphia, PA, USA. ${ }^{6}$ Department of Medical Ethics and Health Policy, Perelman School of Medicine at the University of Pennsylvania, Philadelphia, PA, USA.

\section{Compliance with ethical standards}

\section{Conflicts of interest}

Dr. Halpern is a paid consultant to the American Board of Internal Medicine Foundation's Choosing Wisely campaign.

Received: 7 November 2016 Accepted: 30 November 2016 Published online: 8 December 2016

\section{References}

1. Cutler DM (2015) From the Affordable Care Act to affordable care. JAMA 314:337-338

2. Halpern SD, Becker D, Curtis JR et al (2014) An official American Thoracic Society/American Association of Critical-Care Nurses/American College of Chest Physicians/Society of Critical Care Medicine policy statement: the Choosing Wisely ${ }^{\circledR}$ Top 5 list in critical care medicine. Am J Respir Crit Care Med 190:818-826

3. Luce JM, Rubenfeld GD (2002) Can health care costs be reduced by limiting intensive care at the end of life? Am J Respir Crit Care Med 165:750-754

4. Khandelwal N, Benkeser D, Coe NB, Engelberg RA, Teno JM, Curtis JR (2016) Patterns of cost for patients dying in the intensive care unit and implications for cost savings of palliative care interventions. J Palliat Med 19:1171-1178

5. Belopolskaya OB, Smelaya TV, Moroz W, Golubev AM, Salnikova LE (2015) Clinical associations of host genetic variations in the genes of cytokines in critically ill patients. Clin Exp Immunol 180:531-541

6. Holder AL, Gupta N, Lulaj E et al (2016) Predictors of early progression to severe sepsis or shock among emergency department patients with nonsevere sepsis. Int J Emerg Med 9:10

7. Chang DW, Shapiro MF (2016) Association between intensive care unit utilization during hospitalization and costs, use of invasive procedures, and mortality. JAMA Intern Med 176:1492-1499

8. Valley TS, Sjoding MW, Ryan AM, Iwashyna TJ, Cooke CR (2015) Association of intensive care unit admission with mortality among older patients with pneumonia. JAMA 314:1272-1279

9. Stelfox HT, Hemmelgarn BR, Bagshaw SM et al (2012) Intensive care unit bed availability and outcomes for hospitalized patients with sudden clinical deterioration. Arch Intern Med 172:467-474

10. Wagner J, Gabler NB, Ratcliffe SJ, Brown SE, Strom BL, Halpern SD (2013) Outcomes among patients discharged from busy intensive care units. Ann Intern Med 159:447-455

11. Girard TD, Kress JP, Fuchs BD et al (2008) Efficacy and safety of a paired sedation and ventilator weaning protocol for mechanically ventilated patients in intensive care (awakening and breathing controlled trial): a randomised controlled trial. Lancet 371:126-134

12. Sprung CL, Annane D, Keh D et al (2008) Hydrocortisone therapy for patients with septic shock. N Engl J Med 358:111-124

13. Lakhal K, Macq C, Ehrmann S, Boulain T, Capdevila X (2012) Noninvasive monitoring of blood pressure in the critically ill: reliability according to the cuff site (arm, thigh, or ankle). Crit Care Med 40:1207-1213

14. Moriates C, Soni K, Lai A, Ranji S (2013) The value in the evidence: teaching residents to "Choose Wisely". JAMA Intern Med 173:308-310

15. Green ML, Aagaard EM, Caverzagie KJ et al (2009) Charting the road to competence: developmental milestones for internal medicine residency training. J Grad Med Educ 1:5-20

16. Detsky AS, Verma AA (2012) A new model for medical education: celebrating restraint. JAMA 308:1329-1330

17. Ubel PA, Asch DA (2015) Creating value in health by understanding and overcoming resistance to de-innovation. Health Aff (Millwood) 34:239-244

18. Courtright KR, Weinberger SE, Wagner J (2015) Meeting the milestones. Strategies for including high-value care education in pulmonary and critical care fellowship training. Ann Am Thorac Soc 12:574-578 